\title{
Rethink the immune connection
}

\author{
Recent research suggests that the fight against type 1 diabetes is focusing \\ too narrowly on the adaptive immune system, says Carla Greenbaum.
}

$\mathrm{D}$ ecades ago, investigators established the pathology of type 1 diabetes (T1D) - the adaptive immune system mistakenly attacks the insulin-producing beta cells in the pancreatic islets. Long before the clinical onset of disease (defined by hyperglycaemia), the immune assault triggers a progressive process of beta-cell dysfunction and cell death. As this process unfolds, diabetes-related autoantibodies begin to circulate through the body, and the secretion of insulin is impaired. The attack continues after diagnosis.

This model has served us well in predicting who will get the disease. For example, a relative of someone with T1D who has one of the diabetes-related antibodies has about a 3\% chance of developing T1D over the next five years; those with two or more antibodies have a 35-85\% chance. Although some beta cells remain when clinical symptoms appear, over time the beta cells are completely destroyed.

But an explosion of data about the immune system is yet to yield a cure or prevention strategy for T1D. And we now have the results of several clinical trials testing the hypothesis that it is the adaptive immune system that is wreaking havoc on beta cells. In individuals with recently diagnosed diabetes, altering components of the adaptive immune system, for example through anti-T-cell therapies or anti-B-cell therapies, seems to improve insulin secretion (an indication of betacell function) by roughly $25 \%$ compared to control subjects ${ }^{1}$. Better beta-cell function is associated with important clinical benefits - less hypoglycaemia and fewer complications - but with limited clinical data, the long-term benefits to individual patients remain unknown.

\section{UNSUSTAINABLE RESPONSE}

Moreover, attempts to use short-term treatments to induce longterm immune tolerance of beta cells in a bid to stop disease have not worked. The best interventions so far have slowed the rate of decline of beta-cell function within the first months of diagnosis, but repeated or continued treatments failed to sustain this response. It has also been postulated that treating a T1D patient with antigen, such as insulin or GAD65 in alum, could safely induce tolerance (see 'Cell savers', page S4). However, beta-cell function continues to deteriorate in people with diagnosed diabetes who receive antigen. It is possible that antigen therapy might work at different doses, in different populations of people (particularly earlier in the disease course), and in conjunction with other therapies. And yet, clinical trials testing insulin as a prophylactic whether delivered nasally or parenterally - also failed to prevent diabetes in those who were identified as at risk for type 1 diabetes ${ }^{2,3}$.

One interpretation of these clinical failures is that we have not been aggressive enough in our attempts to save beta cells in those with T1D. An uncontrolled trial of haematopoietic stem-cell therapy to save beta cells has had some success ${ }^{4}$. However, this approach has unknown benefits and is fraught with risks, such as pneumonia and decreased gonad function; just because we can do it, it doesn't mean we should - especially in a disease affecting children.

Rather than not being aggressive enough with therapies, an alternative explanation to the limited success seen to date is that we have narrowly defined therapeutic targets in our intervention trials - namely molecules and pathways of the adaptive immune system. There is undoubtedly a role of the innate immune system and inflammation in beta-cell destruction; clinical trials testing this hypothesis, including blocking the proinflammatory protein interleukin-1, are underway. Moreover, beta cells in T1D might not be the victim of an immune attack, but rather have defective responses to injury or stress. It is true that genome wide association studies (GWAS) have implicated immune related genes. But these same genes have other functions, including influencing beta-cell function and response. When we look at results from an immune-centric approach, we risk missing other factors that can contribute to beta-cell dysfunction. For instance, several hypotheses suggesting that environmental and behaviours factors play a role in the climbing incidence of T1D world wide ${ }^{5}$ await further testing. Our next generation of trials must address multiple components - immunology, genetics, environment and behaviour. Animal models alone will not be enough to guide our future endeavours.

\section{UNSUSTAINABLE RESPONSE}

Before we embark on other large clinical trials, we need more basic research, particularly proof-of-mechanism studies ${ }^{6}$. Such clinical research entails testing new therapeutic approaches in a small number of individuals to measure a biological or mechanistic response. This is the way to examine how alterations in metabolic state or beta-cell stress affect immune function, or to assess off-target effects of combination therapy. Evaluating all data should allow us to guard against evidentiary conservatism (the tendency to base clinical inferences on narrow classes of evidence) and to design the next generation of studies with open minds. To change the course of diabetes, we might need to alter our course.

Carla Greenbaum is an endocrinologist and director of the diabetes program at the Benaroya Research Institute in Seattle, Washington. email: cjgreen@benaroyaresearch.org

\footnotetext{
1. Orban,T. et al. Lancet 378, 412-419 (2011).

2. Diabetes Prevention Trial Study Group. N. Engl. J. Med. 346, 1685-1691 (2002).

3. Nanto-Salonen, K. et al. Lancet 372, 1746-1755 (2008).

4. Voltarelli, J. C. et al. J. Am. Med. Assoc. 297, 1568-1576 (2007).

5. Karvonen, M. et al. Diabetes Care 23,1516-1526 (2000)

6. Kimmelman, J. \& London, A. J. PLoS Med. 8, e1001010 (2011).
} 